RIEF Revue italienne d'études françaises

Littérature, langue, culture

3 | 2013

Varia

\title{
L'enchevêtrement épistolaire du Lys dans la vallée
}

Ilaria Vidotto

\section{OpenEdition}

Journals

Édition électronique

URL : http://journals.openedition.org/rief/230

DOI : 10.4000/rief.230

ISSN : 2240-7456

\section{Éditeur}

Seminario di filologia francese

\section{Référence électronique}

\|laria Vidotto, "L'enchevêtrement épistolaire du Lys dans la vallée », Revue italienne d'études françaises [En ligne], 3 | 2013, mis en ligne le 15 décembre 2013, consulté le 19 avril 2019. URL : http:// journals.openedition.org/rief/230 ; DOI : 10.4000/rief.230

Ce document a été généré automatiquement le 19 avril 2019

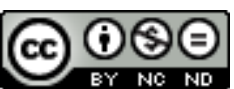

Les contenus de la RIEF sont mis à disposition selon les termes de la Licence Creative Commons Attribution - Pas d'Utilisation Commerciale - Pas de Modification 4.0 International. 


\title{
L'enchevêtrement épistolaire du Lys dans la vallée
}

\author{
Ilaria Vidotto
}

\section{Le discours de l'absence}

1 La lettre est, par définition, le discours de l'absence; sa fonction principale est de véhiculer des informations de toute sorte et de rapprocher, à travers un dialogue à distance et par le biais de l'écrit, deux êtres qui sont éloignés et ne peuvent se parler directement. Les éléments constitutifs de l'écriture épistolaire sont donc essentiellement au nombre de trois : les acteurs de la communication, le rapport qu'ils entretiennent et le canal utilisé. Les deux instances communicatives convoquées par la lettre sont un destinateur, ou locuteur, et un destinataire ou allocutaire. Le destinateur est le sujet dialogique, le « je » qui émet le message destiné à un « tu » ou à un « vous » qui à son tour peut devenir, par sa réaction ou réponse, le sujet émetteur ${ }^{1}$. La relation intersubjective qui s'instaure entre ces deux protagonistes, tout comme leur existence en tant qu'instances de la communication, n'est pourtant liée qu'à l'espace de la lettre, car ils ne subsistent pas en dehors de celle-ci.

2 Le rapport qu'entretiennent les épistoliers est marqué par une dialectique de présence et absence. La raison principale qui généralement nous pousse à écrire une lettre est l'absence physique de l'autre, ce qui nous oblige à recourir à cette forme de «dialogue différé $\rrbracket^{2}$ où la temporalité se trouve dilatée, car le temps de l'écriture ne coïncide jamais avec le temps de la réception. Ce déroulement in absentia de la communication est souvent thématisé par les correspondants à l'intérieur de l'échange même, surtout dans la lettre d'amour, où les amants souffrent de la distance qui les sépare. La tâche essentielle de la lettre est par conséquent de franchir l'espace et de nous rapprocher de l'autre absent ; cela est possible grâce aux pronoms personnels et aux références spatiotemporelles qui contribuent à actualiser l'énonciation et créent l'illusion d'un dialogue avec l'interlocuteur. Toutefois, parallèlement à cette démarche d'actualisation, l'inscription dans la lettre de la situation d'énonciation finit par accentuer l'effet de 
distance, car le décalage temporel séparant le temps de l'écriture de celui de la lecture devient dans la lettre " une modalité d'organisation textuelle ${ }^{3}$ et finit paradoxalement par rendre l'absence encore plus tangible. Cette complémentarité entre présence et absence nous paraît particulièrement intéressante car elle fait de la lettre un instrument précieux pour créer artificiellement une distance et pour placer l'autre dans un lieu aussi protégé qu'inatteignable.

3 L'utilisation du canal écrit comporte également des conséquences non négligeables. À la différence de la parole orale, immédiate et directe, l'écrit offre aux correspondants l'avantage de la préméditation: le message peut être longuement élaboré et poli sans craindre d'impatienter l'interlocuteur; qui plus est, la médiation de l'écrit permet au sujet scripteur de se protéger et de se façonner une image, un ethos discursif calibré en fonction de l'interlocuteur et des effets désirés, toute lettre étant toujours un moyen d'action sur l'autre. Il s'avère donc que les instances épistolaires, même dans le cas de lettres non fictives, sont toujours des figures imaginaires, élaborées en fonction du but à atteindre et dont la construction repose sur des stéréotypes, c'est-à-dire sur des rôles précis et identifiables car fondés sur des compétences culturelles partagées. Le choix des stéréotypes caractérise le style de l'épistolier, influence sa manière de s'adresser à l'autre et constitue selon Anne Jaubert « le trait basique de la connivence »"

4 La lettre se révèle donc moins le simple portrait de l'âme qu'un instrument efficace permettant d'agir, voire même de manipuler l'autre, ou pour le moins de donner une image autre de soi-même. En outre, la simultanéité de présence et absence se développant à l'intérieur de la lettre et la mise à distance de l'autre que permet la parole écrite aident les individus à surmonter l'effet paralysant du face-à-face en donnant l'illusion de pouvoir tout dire.

5 En nous appuyant sur l'analyse de l'épistolarité du Lys dans la vallée, roman de Balzac publié en 1836, nous essaierons de montrer comment les caractéristiques formelles et les potentialités de la lettre brièvement dégagées ci-dessus peuvent être exploitées dans l'écriture de fiction aussi bien au niveau structurel que thématique.

\section{Le Lys dans la vallée : la lettre-confession de Félix}

Le Lys dans la vallée occupe sans doute une place à part dans cet immense édifice littéraire qu'est la Comédie humaine en vertu de son statut hybride. Balzac intègre en effet à une narration rétrospective à la première personne proche des autobiographies fictives quelques artifices propres du roman par lettres (par exemple la présence du cadre péritextuel ou l'inscription du destinataire dans le texte) et fait en outre recours à la technique de la diffraction et de la multiplication des points de vue typique de la forme épistolaire polyphonique. Le récit se présente comme une longue lettre-confession que le protagoniste, Félix de Vandenesse, envoie à sa maîtresse, Natalie de Manerville, à la demande de celle-ci et où il raconte l'histoire de son amour passé (malheureux) pour une autre femme, Henriette de Mortsauf. La structure du roman est assez complexe pour ce qui est des niveaux narratifs : la longue épitre de Félix contient la transcription de quelques lettres de Félix lui-même et d'Henriette, et est encadrée par l'échange épistolaire se composant du billet d'envoi à travers lequel Félix présente sa confession à Natalie et de la réponse de celle-ci. Nous avons donc, suivant la terminologie genettienne, trois niveaux narratifs : un niveau extradiégétique où Félix s'adresse à Natalie et reçoit sa réponse; un niveau diégétique, où se déroule l'intrigue qui a comme protagonistes 
principaux Félix et Henriette et qui intègre un niveau métadiégétique où ces deux personnages commentent ou, plus exactement, « réécrivent » dans leurs lettres certains épisodes-clés du récit. La lettre à Natalie, où Félix étale ses déboires amoureux, constitue donc l'histoire ${ }^{5}$ proprement dite et fournit de nombreuses pistes d'interprétation; notre analyse se bornera à la mise en relief de trois éléments principaux : l'usage de la première personne et le recours au régime de l'aveu, l'emploi de l'écrit par rapport à l'oral ainsi que les obstacles liés au langage et à la communication ${ }^{6}$.

7 Félix entame une démarche de dévoilement de son passé qui assume aussitôt les traits

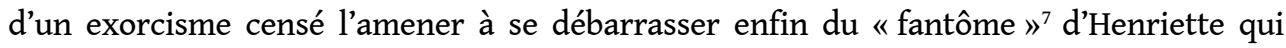
hante son existence. Pour que cela se réalise, il se doit de tout dire, « sans réserve et sans artifice» (p. 384), et s'engage dans la rédaction d'un récit à la première personne, mode par excellence de l'aveu. Cependant, il ne semble pas avoir a priori l'intention de se replier sur son «je» et de conduire un monologue autiste; au contraire, il inscrit le destinataire - quoique celui-ci soit souvent indéterminé - à l'intérieur de sa narration et recherche opiniâtrement le dialogue dans l'espoir d'être compris, voire absous. En effet, si l'aveu est perçu par Félix comme l'arrachement d'un secret, comme l'exhumation insidieuse et certes non volontaire d'« imposants souvenirs ensevelis au fond de [son] âme » (p. 37), c'est qu'il est conscient de sa culpabilité et de ses responsabilités moins visà-vis de sa destinataire actuelle, Natalie, que de celle qui semble véritablement à l'origine de la confession ${ }^{8}$, la défunte Henriette. D'où l'étrange dynamique que L. Frappier-Mazur a mise en lumière : l'aveu de Félix oscille toujours entre «l'auto-accusation et [...] l'autojustification ${ }^{9}$, le premier mouvement étant le cri sincère de la conscience et le deuxième l'étouffement du premier au moyen de l'écriture. Un seul exemple suffira: les justifications pseudo-anthropologiques que Félix apporte, sous forme de prétérition, pour expliquer ce qu'il perçoit comme un crime, à savoir son égarement sensuel entre les bras d'Arabelle («sans vouloir ici justifier mon crime, je vous ferai observer, Natalie, qu'un homme a moins de ressources pour résister à une femme que vous n'en avez pour échapper à nos poursuites ", p. 279).

8 Néanmoins, la recomposition du passé sur le papier n'est pas exempte d'une sorte de jouissance pour Félix : «le plaisir de se confesser et de se connaitre $~^{10}$ semble l'emporter sur la douleur de la confidence, la mise en rapport de sa destinée avec la création des poètes dissocie dès le début l'acte d'écrire de sa fonction informationnelle, si bien que l'exorcisme du passé échoue et celui-ci revient plus vif et attirant que jamais, car purifié par l'écriture ${ }^{11}$. L'aspiration au dialogue se double ainsi d'un narcissisme narratif que Félix n'arrive pas à réprimer et qu'il admet naïvement à la fin du récit : «je fouille ce monceau de cendres et prends plaisir à les étaler devant vous» (p. 381); la lettre devient ainsi une mystification, partant le lieu d'un faux dialogue abritant en réalité la « métaphysique voluptueuse du sujet $~_{12}$ Félix.

9 L'encre et le papier consentent à Félix de ménager la distance spatiale nécessaire à l'épanchement inconditionné qu'il poursuit; l'écriture se présente comme une sorte d'espace protégé où il croit pouvoir tout dire ${ }^{13}$. Cette protection va pourtant bien au-delà de la mise à distance du destinataire, étant donné que la rédaction d'une lettre ne peut jamais être séparée d'une fictionnalisation du sujet écrivant qui s'opère par la construction d'une image de soi non authentique. Or, Félix semble tirer profit de ce mécanisme, car la verbalisation de ses souvenirs transfigure l'expérience vécue et lui permet non seulement de façonner sa propre identité d'amoureux tourmenté, mais de donner aussi une identité à Henriette et de choisir le mode de leur relation. Nous 
rappelons à ce propos la célèbre scène du bal de Tours, où Félix rencontre pour la première fois Madame de Mortsauf; c'est bien le moment qui déclenche l'action et à partir duquel le héros-narrateur forge l'imagerie de la mère et de l'enfant (« une femme me prit pour un enfant prêt à s'endormir en attendant le bon plaisir de sa mère et se posa près de moi », p.60) ainsi que le code angélique qui va moduler ses rapports avec Henriette ${ }^{14}$ tout au long de la narration, dans le but d'atténuer - voire même de refouler une tension érotique incontournable. Dès lors, Henriette restera figée dans l'habit de sainte éthérée, de mère angélique que Félix va coudre grâce aux mots, et acceptera, non sans complaisance, le jeu de rôles bizarre que les amants s'infligent pour inscrire leur passion dans la sphère de ce qui est permis : la mère et l'enfant, le frère et la sœur, autant de masques qui nient le corps et cachent l'indicible du désir.

Ce manque de franchise se rattache à l'autre thème fondamental du récit de Vandenesse que Victor Brombert appelle « le drame du langage $»^{15}$ et qui s'inscrit, à nos yeux, dans le cadre plus vaste d'un échec de la communication. On a rappelé que la relation entre Félix et Henriette se fonde sur un interdit du désir qui correspond à un interdit de parole ; pour les amants, le dire est dramatiquement un faire, ils perçoivent les mots comme hyperperformatifs et dès les premières confidences Félix évite le mot " amour » car il renvoie à un sentiment «qu'[Henriette] ignore et qui ne [lui] est point permis» (p.132). Pour contourner les embûches d'une parole menaçante, les amants recourent à un code oblique et imprégné de mysticisme, à de nombreuses médiations comme la métaphore de l'enfance ou le langage des fleurs ${ }^{16}$. Or, ce code est en réalité bien loin de la neutralité et de l'innocence, et les deux en sont conscients car ils jouissent d'un "contentement semblable à celui de l'esclave qui trompe son maitre » (p. 167). La tromperie se révèle toutefois une arme à double tranchant : en croyant tromper le désir par le bannissement de la parole, ils ne font que l'aiguiser et finissent par se tromper eux-mêmes, car la nature ne se laisse pas dompter et, comme le constate amèrement Henriette, « le ciel et la terre sont incompatibles » (p. 311). Ce n'est que dans les quelques lettres qu'ils s'écrivent - et qui sont reproduites à l'intérieur du récit - que les amants essaient de se réapproprier le langage ordinaire et de surmonter les tabous d'une communication in presentia minée par l'interdit, même si la distance ménagée par l'écrit ne suffit pas à elle seule pour rétablir la franchise entre eux.

11 Voilà donc quelques-uns des nombreux jalons qui structurent la lettre de Félix, dont la volonté de tout dire se heurte en réalité à un manque fondamental de sincérité et à une non-acceptation du passé, de sorte qu'il commet avec Natalie, au niveau extradiégétique, la même erreur que celle qu'il a commise avec Henriette. Si une communication ouverte avait été jadis absolument impossible à cause des interdits et des ambiguïtés que les amants s'étaient imposés, l'épanchement libre auquel Félix aspire dans sa lettreconfession s'avère également utopique. Félix ne parvient pas à garder une distance critique vis-à-vis du passé et finit par dresser un portrait à la fois mythique et mystifié de son amour pour Madame de Mortsauf, dont le fond et la forme ne peuvent que froisser sa maitresse actuelle, qui rejette sans appel l'ethos discursif du narrateur.

\section{Le cadre : le billet d'envoi de Félix et la réponse de Natalie}

Les deux lettres qui encadrent le corps de la narration - et qui constituent de fait le seul véritable échange épistolaire du roman - jouent un rôle de premier plan pour la 
complétude structurelle du texte ${ }^{17}$, puisqu'elles permettent entre autres à Balzac de reprendre en dehors de l'histoire proprement dite certains sujets abordés en creux dans le récit lui-même, à savoir l'échec de la communication et le rapport souvent controversé entre lecteur et auteur.

Le message liminaire de Félix recouvre en quelque sorte la fonction d'une préface car il annonce ce qu'on va lire (« aujourd'hui tu veux mon passé, le voici » p. 38) et précise la relation que l'émetteur de la lettre entretient avec son destinataire; Félix fait en effet allusion à la demande de Natalie qui est à l'origine de la confession et propose à son amante un pacte épistolaire que nous pouvons résumer ainsi : le dévoilement de son passé en échange d'un amour sans conditions. L'inscription du narrataire, marquée par les nombreux adjectifs et pronoms à la deuxième personne du singulier, contribue à mettre en place le dialogisme qui caractérisera aussi le ton de la confession et qui est renforcé par quatre interrogations et par des verbes à l'impératif. Nous sommes en présence d'un dialogue entamé dans une situation antérieure non précisée et qui paraît tronqué, car nous lisons les réponses (« je cède à ton désir »; " oui, ma vie est dominée par un fantôme ", p. 37) mais nous devons reconstruire les questions.

Ce schéma de question-réponse nous introduit d'emblée dans une atmosphère judiciaire où Félix fait figure d'accusé au banc des témoins, contraint de dévoiler ces «imposants souvenirs ensevelis au fond de [son] âme " pour satisfaire le désir d'un destinataire tyrannique et capricieux qui n'a probablement pas assez mesuré le danger intrinsèque à toute confidence. Bien que le régime de l'aveu le mette en position de nette infériorité ${ }^{18}$ par rapport à Natalie, Félix semble procéder à une espèce de dédouanement au préalable : en se retranchant derrière le stéréotype de matrice courtoise du chevalier qui obéit à la volonté de sa dame, il présente ce qui va suivre comme un aveu extorqué par l'insistance curieuse de Natalie et tient surtout à se déresponsabiliser vis-à-vis des conséquences pour le destinataire (" s'il y avait dans cette confession des éclats qui te blessassent », p. 38). La résurrection sur le papier d'Henriette, ce «fantôme » qui n'a jamais cessé de hanter sa vie, a en effet produit des réactions inattendues chez un Félix subjugué malgré lui par l'emprise nostalgique du passé et qui est donc d'autant plus conscient du risque que comporte la rédaction du billet après la confession. Il essaie ainsi de mettre en garde Natalie contre un danger qu'elle-même a créé, mais la mise en garde se double aussitôt d'une certaine agressivité témoignant de son amertume et de son irritation pour ne pas avoir su défendre son secret. Par l'emploi des verbes vouloir et céder, par les avertissements à l'impératif, l'allusion aux menaces qui ont accompagné la requête (" tu m'as menacé si je ne t'obéissais pas ", p. 38) et aux punitions qui pourraient suivre malgré son obéissance, Félix dresse un portrait impitoyable de sa despotique amie et entreprend de fait une démarche qui révèle indirectement aussi bien sa faiblesse que ses tiraillements entre la contrainte du dire et le regret d'avoir finalement vaincu les « répugnances inviolées » (p. 37) qui l'ont retenu jusque-là. Qui plus est, le topos de l'arrachement du secret découle aussi du besoin souterrain de se rendre intéressant, de trouver un fairevaloir adéquat pour son récit, ce qui fait poindre en creux le mouvement d'orgueil typique de tout auteur ainsi que le sujet très sensible pour Balzac lui-même du rapport souvent difficile entre écrivain et lecteurs.

15 La lettre de réponse de Natalie suit directement la fin de la longue confession de Félix et marque la rupture du temps diégétique avec le retour à la dimension extradiégétique où se situe aussi le billet d'envoi. Le lecteur est quelque peu surpris de trouver, en guise de clôture, la réponse à ce billet qu'on avait presque oublié ; les trois cents pages de pseudo- 
monologue de Félix ont en effet différé la communication épistolaire entre les deux partenaires et mis en danger un dialogue troublé dès le début par le spectre de l'incompréhension. S'agissant d'une lettre, donc d'une communication médiatisée, Natalie peut procéder dans l'exorde à la définition de son ethos de femme mondaine qui, froissée par la maladresse de son amant, s'applique à lui apprendre les règles du monde (elle se doit, dit-elle, «d'achever [son] éducation » p. 386) et ne lui fait pas grâce de son sarcasme poignant. Les griefs, par lesquels Natalie semble vouloir reprocher au prétendu poète Félix son inélégance, découlent moins du désir mesquin de revanche que d'une lecture très précise de la confession de son amant. En assumant aussitôt une posture de lectrice attentive ("après avoir lu votre récit avec l'attention qu'il mérite», p. 386) qui s'accorde le droit de critiquer l'auteur et de faire des remarques ponctuelles, elle propose une version inédite des faits et ajoute un autre éclairage sur une histoire qu'on avait jugée digne de foi sur la base de la narration à la première personne et l'identité entre auteur et personnage.

16 Natalie oppose une fin de non-recevoir brutale à la requête d'indulgence de son amant et déconstruit de bout en bout le «bel édifice » (p. 381) de Félix; elle pointe le déchirement coupable d'un homme qui, au lieu de faire un choix entre Henriette et Arabelle, n'a fait que poursuivre la chimère d'une union impossible entre « l'eau et le feu » (p. 388), et qui a fini par méconnaitre la vraie nature des deux femmes: "vous avez rencontré Lady Dudley trop tôt pour pouvoir l'apprécier [...] vous avez compris madame de Mortsauf trop tard» (p. 388). Mais c'est surtout envers Natalie elle-même que Félix a le plus « manqué de tact» (p. 386). En tant que lectrice vigilante, elle a certainement saisi, tout comme le lecteur extradiégétique, les clins d'œil peu délicats de Félix, qui semble avoir pris le soin de la faire apparaitre inférieure en vertus et en beauté par rapport à la "sainte Henriette»(p. 386) ${ }^{19}$. Elle l'accuse surtout de l'avoir sans cesse contrainte à une comparaison avec deux fantômes qu'il ne semble finalement pas vouloir chasser et qui "forcent une femme à douter d'elle-même" (p.387), de sorte qu'elle refuse catégoriquement ce mélange trouble de passé et de présent que Félix a essayé de réaliser par l'écriture. Elle foule aux pieds le « programme inexécutable » (p. 388), la synthèse que le récit lui proposait (« être à la fois madame de Mortsauf et lady Dudley [...] n'est-ce pas vouloir réunir l'eau et le feu? » p. 388) ainsi que le pacte épistolaire que Félix avait établi dans le billet d'envoi et réaffirmé à la fin de son récit. Natalie revendique ainsi son autonomie de femme et de lectrice et rejette également le style amphigourique et nourri de stéréotypes de Félix; les virtuosités et les images ampoulées sont rapetissées au rang de "phrases sentimentales» (p. 388) agencées non par un poète voyant ${ }^{20}$ mais par un monstre qui a fait « mourir de chagrin » (p. 387) sa bien-aimée et qui cherche maintenant à se racheter en lui édifiant « un tombeau littéraire » étouffant ${ }^{21}$.

17 Sa critique n'épargne donc ni le fond, ni la forme, ni surtout le choix de l'écrit par rapport à l'oral : en se servant de l'écran de la lettre, Félix a cru pouvoir tout dire comme s'il parlait à lui-même et non à un destinataire qu'il faut ménager, quitte à renoncer à la sincérité et à la complétude. « Il fallait me tromper » (p. 388), écrit Natalie, et elle atteste ainsi l'échec amoureux et littéraire d'un Félix hanté par le fantôme de l'impossible franchise et qui a confondu la morte et la vivante en croyant pouvoir charmer la seconde par un style qui convenait mieux à la première. Natalie ne partage pas le même code et cela entraîne le naufrage de leur dialogue, en montrant aussi que l'espace épistolaire est, dans Le Lys dans la vallée, le lieu d'une communication non aboutie. 
18 Le personnage de Natalie, bien que périphérique et de fait immatériel, joue donc un rôle essentiel dans l'économie du roman, car elle incarne aussi et surtout la figure du lecteur que nous sommes; ceci permet à Balzac de représenter en abyme l'acte de lecture et de se préfigurer les réactions potentielles de ses lecteurs, qu'il cherche à prévenir par une "précaution auto-critique, [...] une ironie protectrice ${ }^{22}$ révélant sa hantise - qui est aussi celle de Félix - de ne pas être compris.

\section{Les lettres à l'intérieur du récit}

19 Les lettres qui émaillent le récit du Lys dans la vallée et qui constituent les traces les plus évidentes du renouement de Balzac avec la tradition épistolaire du XVIIIe siècle sont au nombre de trois : la lettre d'amour que Félix envoie à Madame de Mortsauf, la lettreviatique de celle-ci à Félix et l'aveu final d'Henriette.

20 La première missive de Félix marque un moment décisif de l'action, car elle se situe après la scène dramatique où Mme de Mourtsauf s'évanouit suite à la terrible dispute avec son mari et qui occasionne le premier contact physique entre les amants. Se retrouvant seul dans la chambre d'Henriette, en proie à une émotion paralysante, Félix ne trouve pas les mots pour conforter la souffrante et fait donc recours à la lettre, qui s'avère le seul canal licite pour exprimer les sentiments qui l'agitent et pour surmonter les impasses de l'oral ; néanmoins, il prend conscience de l'insuffisance du langage et du décalage énorme séparant, même à l'écrit, le ressenti et le dit (« des trois, quatre lettres que je fis, il m'est resté ce commencement dont je ne fus pas content ; [...] il me parut ne rien exprimer, ou trop parler de moi quand je ne devais m'occuper que d'elle » p. 189), ce qui peut aussi à la longue peser sur la communication. Le passage impromptu du « vous » respectueux mais chargé de tendresse du début de la lettre au «tu » du milieu et de la fin exprime le désir du jeune homme d'atteindre une intimité au-delà des contraintes de la réalité, ce qui ne peut pourtant se réaliser que dans l'espace clos de la lettre, car on imagine déjà que Félix ne réussira jamais à franchir le seuil d'un aveu direct. La parole écrite devient donc pour lui le moyen de "substituer la fiction au réel $»^{23}$ et de rattraper l'occasion perdue auprès d'Henriette de lui dire son amour. Ainsi Félix semble nous dire que seul l'établissement d'une distance factice par le biais de la lettre peut éliminer les obstacles qui entravent le dialogue. Tel est le paradoxe des amants du Lys: avoir élaboré un mode de communication où les notions de présence et d'absence deviennent arbitraires et où la vraie proximité n'est possible que dans l'enveloppe protectrice de l'éloignement. L'absence se révèle finalement la condition nécessaire et suffisante pour l'épanchement ( «je ne peux donc vous parler de vous que loin de vous» p. 189) sans pour autant entraîner l'établissement d'une zone franche - dans tous les sens du terme - à l'abri des interdits; la réponse d'Henriette, ce «ne m'écrivez plus ainsi » (p. 192) plein d'émotion, vient en effet étouffer la fragile tentative de Félix et ne laisse aux amants que les consolations et les faux-fuyants d'une parole médiatisée nourrie de codes et de symboles ${ }^{24}$ qui les expose aux risques du malentendu.

21 La lettre qu'Henriette remet à Félix peu avant le départ de ce dernier pour Paris marque un autre tournant de l'intrigue, à savoir la première séparation des amants, et revêt par conséquent un rôle majeur, comme l'indiquent aussi sa longueur (dix-sept pages) et son ton solennel. En définissant la posture que Félix doit assumer lors de la lecture (il s'agit en effet pour elle de "donner quelques enseignements de mère à fils", p. 196) et en anticipant les sujets de sa missive (« ses pensées de femme sur le monde, sur les hommes, 
sur la manière d'aborder les difficultés dans ces grands remuement d'intérêts ", p. 196), Henriette s'empresse de la situer dans l'espace balisé de la métaphore mère-enfant dans le but de conditionner le mode d'interprétation de Félix. L'étrange et ferme requête: «promettez-moi de ne la lire qu'à Paris?» (p. 196) et ce besoin de creuser une distance entre eux relèvent certes d'une tactique visant à aiguiser la curiosité de son pupille, mais soulignent surtout la volonté de Madame de Mortsauf de se mettre à l'abri de la réaction impétueuse de Félix qui n'aurait pas tort de voir dans ce viatique une lettre d'amour mal déguisée.

Le schéma d'énonciation et les rôles qui se mettent en place ici sont à bien des égards ceux de la lettre didactique. Henriette assume la posture du mentor en train de dispenser de précieux conseils à son élève et étaye ses arguments à l'aide d'exemples clairs, tirés de la vie pratique (« quand notre pauvre homme de la Rhétorière vient se coucher fatigué de ses labours », p. 205), de la littérature («le galant homme est aussi loin de la lâche complaisance de Philinte que de l'âpre vertu d'Alceste », p. 207) ou encore de situations bien connues de Félix («Vous savez combien M. de Mortsauf haïssait Napoléon », p. 213 ), qui doivent lui permettre de mieux suivre le fil du raisonnement. Le ton didactique est renforcé par l'emploi presque exclusif de l'impératif, donnant lieu parfois à des tournures quelque peu péremptoires ("je veux que vous soyez ainsi, Félix», p. 207; «je vous recommande », p. 211), et surtout par les trois devises qu'Henriette forge et qui doivent se graver à jamais dans l'esprit du disciple : «noblesse oblige » (p. 211), « les servir toutes, n'en aimer qu'une» (p. 217) $)^{25}$ et ce «veuillez donc!» qui doit sous-tendre toutes ses actions.

Et pourtant, ces sages conseils, qui se distinguent par une extraordinaire clairvoyance et une grande connaissance des rouages de la société parisienne, ne cachent pas une émotion vibrante. Les interpellations nous montrent déjà le glissement du ton du pédagogique vers le sentimental : Félix est à la fois "mon ami ", "cher Félix», "cher enfant de mon cœur » ; mais c'est surtout dans la dernière partie de la lettre qu'Henriette finit par mettre à nu ses sentiments. En abordant le sujet de sa conduite auprès des femmes, elle conjure Félix de ne cultiver que «les femmes influentes» (p. 214) qui peuvent favoriser sa carrière, tandis qu'il doit impérativement fuir les jeunes femmes, " égoïstes et petites» (p. 215) qui « dévoreront sans scrupule [son] temps, [lui] feront manquer [sa] fortune, [le] détruiront de la meilleure grâce du monde » (ibid.). Une seule femme aura en revanche le droit d'être pour lui «le monde entier» (p. 216) : Henriette la présente comme "la créature chaste et recueillie» (ibid.), solitaire dans son amour, complètement dévouée ( cette femme ne sera jamais elle, elle ne devra jamais penser à elle, mais à vous ", ibid.), et douée d'un regard attentif qui « saura flairer pour vous un danger là où vous n'en verrez point " (ibid.). Ne sommes-nous pas en présence d'un autoportrait passionné ? Malgré l'artifice de la troisième personne, Henriette ne fait que parler d'elle-même, comme le démontre son aveu : « oui, je vous porte une affection dont l'étendue ne vous sera jamais connue» (p. 217), phrase qui nous paraît d'ailleurs être un lapsus métalinguistique, car rien n'est plus saisissant et saisissable dans cette lettre que l'étendue de son affection.

24 Le souci que montre Henriette à propos de la réception et du sens que Félix pourrait donner à ses mots («Suis-je suspecte en vous disant d'éviter les jeunes femmes », p. 217) nous révèle par ailleurs qu'elle a finalement échoué dans sa tentative de camoufler ses sentiments. Au contraire, la conscience de s'être abandonnée à l'expression de son amour et d'avoir dit plus que ne l'était son intention de départ l'oblige à recourir à plusieurs 
reprises à l'écran de la métaphore mère-enfant, ce qui transforme cette lettre en un leurre, en instrument « de manipulation du lecteur ${ }^{26}$ et donc de Félix, lequel n'y sent que les palpitations « d'un cœur maternel» (p. 218).

La dernière lettre qui attire notre attention se situe à la fin du roman et suit la série de rebondissements qui ont entraîné Félix à rompre, dans les bras de Lady Dudley, le vœu de chasteté et de vertu scellé tacitement avec Henriette. Celle-ci, après avoir appris la trahison, tombe dans un "abattement» (p. 291), signe extérieur d'une maladie dévastatrice qui commence à la ronger et qui apparaît surtout comme le dernier sursaut de son corps trop longtemps meurtri, qu'elle laisse enfin parler dans la lettre léguée à Félix en guise de testament. Son effet est d'autant plus retentissant qu'il s'agit d'un discours libéré par l'éloignement définitif, comme si Henriette avait eu besoin «de l'imminence de la mort pour aller jusqu'au bout de sa vérité $\aleph^{27}$. Encore une fois, aucun échange, aucune réplique ne sont possibles et telle semble être, ici comme ailleurs dans le roman, la condition perverse de l'aveu ${ }^{28}$. Henriette semble animée principalement par le désir de donner sa version des faits, de raconter leur relation de son point de vue ; elle procède ainsi, dans le corps de la lettre, à une réécriture de l'histoire qui commence là où tout à commencé, au bal de Tours, et donne une interprétation tout à fait différente de cet épisode. Si le narrateur s'évertue à inscrire ses baisers criminels dans le cadre ambivalent d'une pulsion qui est premièrement désir d'affection maternelle, la comtesse se dit frappée par la vague soudaine d'un désir tout viril éveillant le sien, « qui sommeillai [t] à [son] insu» (p. 372). L'ardeur contagieuse de ces baisers déclenche chez elle le mécanisme inverse par rapport à Félix, puisqu'il identifie en elle une figure maternelle au moment où elle ne se sent "plus mère qu'à demi $»^{29}$. Elle nomme donc ici le gros malentendu qui sous-tend tout le récit: si le texte autorise à lire l'amour maternel d'Henriette comme " le registre passionnel le plus impétueux " ${ }^{30}$, il est tout aussi vrai que la métaphore de l'enfant est utilisée souvent comme écran derrière lequel se cachent les pulsions inavouables d'une femme qui découvre pour la première fois "l'impérieuse volupté » (p.372) dans toute sa puissance. Ainsi, le reproche le plus poignant qu'elle adresse à Félix est non de l'avoir trahie mais de l'avoir emprisonnée dans cette figure d'ange sans sexe, d'âme dépouillée de sa chair, et de ne pas avoir su interpréter les signaux du corps: "si [...] vous m'eussiez prise dans vos bras, je serais morte de bonheur » (p. 372). Connaît-on un démenti plus flagrant pour un narrateur qui avait opiniâtrement affirmé qu'elle « ne connaissait rien ni des plaisirs de l'amour ni de ses tempêtes » (p. 256) ?

Natalie n'est pas la seule à rejeter sans appel le récit de Félix: Henriette avant elle, quoique sur un ton plus bienveillant, renverse complètement la perspective qui avait jusque là orienté la lecture et déconstruit de bout en bout son personnage en rétablissant une vérité qui n'est pas la seule mais qui est au moins la sienne. La dimension polyphonique du roman est ainsi renforcée par ce nouvel éclairage ; il faut pourtant bien se garder de conclure qu'Henriette renonce par cet aveu à la vertu dont elle s'était fièrement parée, car cela reviendrait à méconnaître le vain combat qu'elle a mené contre elle-même et contre le remords de se savoir "moins mère, moins honnête femme " (p. 374), ce qui d'ailleurs l'a poussée à vouloir se dépasser dans son abnégation. Cette figure si complexe et contradictoire nous donne ainsi la mesure du talent romanesque de Balzac qui, au lieu de répéter le schéma un tant soit peu abusé de l'héroïne victime sacrificielle de la vertu, dessine un personnage bien plus riche, à la fois authentiquement 
vertueux et authentiquement sensuel, qui succombe pour avoir compris que le corps ne peut pas être nié et que ses raisons sont aussi fortes que celles de l'âme.

La réaction de Félix à la lecture de la lettre est pour le moins surprenante. Comme l'affirme Raymond Mahieu, « ces pages ont sur leur destinataire une remarquable absence de retentissement $»^{31}$ : Félix ne fait aucun commentaire sur le contenu et se montre plutôt indifférent face à ce qui, pour le lecteur, a la portée d'une révélation. Cette indifférence est le mouvement spontané de quelqu'un qui, voyant son univers représentationnel complètement ébranlé, refuse l'évidence et décide d'ignorer une réalité pour lui inacceptable. La lettre d'Henriette crie pourtant tout haut la vérité du corps et brise «la tyrannie du récit sur le personnage $\aleph^{32}$, en obligeant le lecteur à un processus de réinterprétation assez déstabilisant et en mettant en branle le mécanisme de l'aveu qui gouverne tout le récit, car la confession de Félix peut aussi être lue comme la tentative ingénue de reconstituer sur le papier cette femme aux contours mythiques que la mort lui a doublement arrachée ${ }^{33}$.

\section{Conclusion}

28 Les lettres se révèlent, dans Le Lys dans la vallée, porteuses d'enjeux thématiques importants, aussi bien au niveau diégétique qu'extradiégétique. La polyphonie que créent le cadre péritextuel ainsi que les lettres insérées dans le récit, et notamment l'aveu final d'Henriette, permettent à Balzac de développer en creux la problématique du rapport entre écrivain et public à travers une mise en abyme des actes de lecture et d'écriture. Henriette et Natalie fournissent en effet deux lectures opposées des événements par rapport à Félix, et si la lettre d'Henriette, se situant encore à l'intérieur de l'histoire, a essentiellement l'effet d'un démenti mettant en doute la crédibilité du narrateur, Natalie incarne en revanche la figure du Lecteur qui relève les contradictions de l'auteur (de tout auteur) et revendique son droit à la critique et à l'autonomie d'interprétation.

L'épistolarité révèle en outre les difficultés du dire et l'impossibilité de la parole. Le dialogue entre Natalie et Félix échoue car ce dernier utilise la lettre comme prétexte pour faire nostalgiquement revivre un passé où le destinataire n'a aucun rôle et pour donner libre cours à son narcissisme d'écrivain manqué. Les lettres qu'Henriette et le narrateur s'envoient installent entre eux une distance artificielle qui permet certes de surmonter les impasses du face-à-face, mais qui ne débouche pas sur un échange épistolaire établi, vu que les amants semblent tout de même préférer l'écran d'un langage médiatisé qui, en éludant les mots, les met à l'abri de leurs pièges. Ainsi, le paradoxe flagrant que nous montrent les lettres - dont le but serait a priori de nous rapprocher de l'absent - est que l'éloignement et l'absence sont les conditions essentielles pour entamer un semblant de dialogue : Félix écrit à Henriette car les mots s'évanouissent en sa présence et Henriette attend l'enveloppe protectrice de la mort pour crier enfin son désir.

En nous invitant à réfléchir sur les impasses de la communication et en dynamisant la narration à travers une pluralité de voix et d'angles d'interprétation, les lettres contribuent à faire du Lys un roman qui, comme tous les grands romans, "gagne à être relu $\|^{34}$. 


\section{NOTES}

1. Pour une analyse plus approfondie des rôles et des instances intervenant dans la lettre voir aussi A. J. Greimas (dir.), La Lettre: approches sémiotiques. Les actes $d u \mathrm{VI}^{e}$ colloque interdisciplinaire, Fribourg, Éditions universitaires de Fribourg, 1988, et J. Siess (dir.), La lettre entre réel et fiction, Paris, SEDES, 1998.

2. P. Violi, «Présence et absence. Stratégies d'énonciation dans la lettre », dans A. J. Greimas (dir.), op. cit., p. 27.

3. Ibid., p. 32.

4. A. Jaubert, «La correspondance comme genre éthique », Argumentation et analyse du discours, 5, 2010, <http://aad.revues.org/985>, consulté le 3 mai 2012.

5. Cf. G. Genette, Palimpsestes, Paris, Éditions du Seuil, 1982, p. 342.

6. Selon Isabelle Dominati-Baruchel, le choix d'une confession écrite témoignerait du narcissisme mal caché de Félix et de sa volonté de fuir la confrontation directe avec Natalie - d'où la punition que cette dernière lui réserve à la fin du roman. $C f$. I. Dominati-Baruchel, « Aimer ou écrire : de la lettre au roman », dans J.-L. Diaz (dir.), Balzac, Le Lys dans la vallée, "cet orage des choses célestes", Paris, SEDES, 1993, p. 55-64.

7. H. de Balzac, Le Lys dans la vallée, Paris, Le livre de poche, «Classiques de poche», 1995, p. 38. Toutes nos citations renvoient à cette édition.

8. Nicole Mozet avance que Félix «profite de l'ambiguïté de l'écriture pour répondre à la lettre posthume d'Henriette tout en s'adressant à Natalie », dans Préface au Lys dans la vallée, Paris, Flammarion, 2010, p. 23.

9. L. Frappier-Mazur, « Le régime de l'aveu dans Le Lys dans la vallée. Formes et fonctions de l'aveu écrit », dans Revue des sciences humaines, 175, 1979, p. 7.

10. Ibidem.

11. "Le Lys est aussi un roman de l'écriture, avec cette longue lettre qui est supposée répondre au désir amoureux de Natalie et qui ne comble que le désir de Félix d'écrire, sous le prétexte de tout dire ", I. Dominati-Baruchel, op. cit., p. 59.

12. A. J. Greimas, Préface à «La Lettre»-approches sémiotiques, op. cit., p. 7.

13. I. Dominati-Baruchel parle à ce propos du «fantasme de la communication totale » que poursuit Félix et que seul le rejet sans appel de Natalie dissipera, op. cit., p. 57.

14. Pour une lecture détaillée de l'épisode, nous renvoyons à R. Mahieu, «Les lapsus du Lys dans la vallée ", dans J.-L. Diaz (dir.), op. cit., p. 97-105.

15. V. Brombert, "Natalie ou le lecteur caché de Balzac", dans Mouvements premiers. Études critiques offertes à Georges Poulet, Paris, Corti, 1972, p. 180.

16. L'épisode des bouquets est emblématique : Félix trouve un exutoire pour son désir dans la composition de deux bouquet luxuriants, où le blanc et le bleu - couleurs mariales par excellence - se mêlent au rouge du pavot symbolisant le débordement de la passion, de sorte qu'ils constituent un langage alternatif et déchiffrable seulement pour les amants. Les bouquets font ainsi l'office d'une lettre où tout peut être dit, même les pulsions les plus violentes.

17. L'analyse des nombreuses versions qui ont précédé la version définitive de 1836 montre que le manuscrit ne contenait à l'origine que la confession de Félix et une seule lettre, celle qu'il écrit à Henriette ; le billet d'envoi et la lettre d'Henriette sont le fruit d'ajouts ultérieurs et ce n'est qu' à la veille de la publication que Balzac insère la réponse de Natalie. Cela démontre l'importance croissante que la dimension épistolaire assume au fur et à mesure que Balzac relit et corrige ses 
épreuves. Pour une description de l'évolution du texte, voir l'appendice critique et les commentaires de G. Séginger dans notre édition, p. 393-474.

18. Cf. L. Frappier-Mazur, op. cit., p. 7.

19. Les exemples sont nombreux : on ne citera ici que les plus voyants, qui ont frappé Natalie elle-même : Félix affirme à la fin du récit que « la plupart des femmes qui montent à cheval ont peu de tendresse ", en oubliant bizarrement que Natalie aussi monte à cheval (p. 339) ; mais l'indélicatesse la plus grave tient aux «bouquets enivrants» (p.387) que Félix n'a plus faits «pour personne » (p. 167) et qu'il refuse aussi à Natalie.

20. Félix s'attribue dès l'enfance des qualités de voyant qui le rapprochent du génie: «J'ai souvent attribué ces sublimes visions à des anges chargés de façonner mon âme à de divines destinées, elles ont doué mes yeux de la faculté de voir l'esprit intime des choses [...] elles ont écrit dans ma tête un livre où j'ai pu lire ce que je devais exprimer [...] » p. 48.

21. G. Séginger, Introduction au Lys dans la vallée, op. cit., p. 12.

22. V. Brombert, op. cit., p. 178.

23. M.-C. Grassi, Lire l'épistolaire, Paris, Dunod, 1998, p. 130.

24. Nous rappelons ici seulement le code mystique, car il colore aussi cette lettre d'amour: Henriette est transfigurée en créature "dégagée des entraves corporelles » où seule l'âme est « visible et palpable » et qui fait comprendre à Félix « l'amour divin, cet amour sûr qui, plein de sa force et de sa durée, ne connaît ni soupçons ni jalousies » (p. 191-192).

25. Il s'agit d'un "mot de chevalerie », comme le dit Henriette elle-même (p. 217), qui renforce les modulations courtoises et moyenâgeuses parsemées dans le discours des amants, l'un des nombreux codes langagiers dont les deux font usage.

26. J. Przybos, "L'épistolarité déchiffrée: Étude sur Le Lys dans la vallée de Balzac", Modern Language Studies, 2, 1986, p. 63.

27. L. Frappier-Mazur, op. cit., p. 10.

28. Notre interprétation diffère ici de celle d'Isabelle Dominati-Baruchel, pour qui cet échange épistolaire proche de la monodie - car les lettres restent toutes sans réponse - permet à Félix et à Henriette d'atteindre « une sorte de perfection de la communication, dans laquelle ne subsiste aucune entrave, aucune retenue, aucun contresens ", I. Dominati-Baruchel, op. cit., p. 58. Or, à notre avis le manque de confrontation franche et de réaction directe de la part du destinataire ne peut qu'augmenter le risque d'incompréhension, voire même entraîner l'échec de la communication.

29. R. Mahieu parle très justement d'une « divergence d'effets liée à l'éviction chez la femme, à l'invocation, chez l'homme, de la référence maternelle ", op. cit., p. 102.

30. V. Dufief-Sanchez, Philosophie du roman personnel de Chateaubriand à Fromentin (1802-1863), Genève, Droz, 2010, p. 277.

31. R. Mahieu, op. cit., p. 100.

32. Ibidem.

33. La mort d'Henriette est double en ce que l'image qu'en avait Félix meurt également, détruite par l'aveu de l'héroïne.

34. H. de Balzac, Lettres à Madame Hanska, t. I, Paris, Robert Laffont, 1990, p. 355. 
INDEX

Mots-clés : communication (schéma de la), écriture épistolaire, dialectique présence-absence, Balzac (Honoré de), Lys dans la vallée, confession, communication (échec de la), niveaux diégétiques, roman polyphonique, mise en abyme du lecteur 